\title{
Large diffractive/refractive apertures for space and airborne telescopes
}

Howard A. MacEwen, James B. Breckinridge

Howard A. MacEwen, James B. Breckinridge, "Large diffractive/refractive apertures for space and airborne telescopes," Proc. SPIE 8739, Sensors and Systems for Space Applications VI, 873904 (21 May 2013); doi: $10.1117 / 12.2015457$

Event: SPIE Defense, Security, and Sensing, 2013, Baltimore, Maryland, United States 


\title{
Large diffractive/refractive apertures for space and airborne telescopes Howard A. MacEwen ${ }^{* a}$, James B. Breckinridge ${ }^{b}$

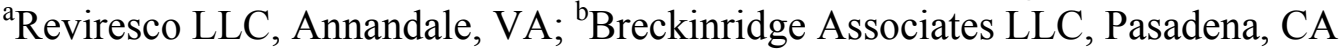

\begin{abstract}
Recent work, specifically the Lawrence Livermore National Laboratory (LLNL) Eyeglass and the DARPA MOIRE programs, have evaluated lightweight, easily packaged and deployed, diffractive/refractive membrane transmissive lenses as entrance apertures for large space and airborne telescopes. This presentation describes a new, innovative approach to the theory of diffractive and refractive effects in lenses used as telescope entrance apertures and the fabrication of the necessary large membrane optics. Analyses are presented to indicate how a broadband, highly transmissive diffractive / refractive membrane lens can be developed and fabricated, and potential applications in defense and astronomy are briefly discussed.
\end{abstract}

Keywords: Diffractive optics, refractive optics, membrane aperture, airborne telescope, space telescope

\section{INTRODUCTION}

\subsection{Large membrane diffractive optics projects}

Diffractive optics has been understood and demonstrated (in the laboratory and small device applications) for over a century, as briefly discussed in the Appendix. Recent significant projects addressing larger system applications and accessible in the public domain include the Lawrence Livermore National Laboratory (LLNL) Eyeglass ${ }^{1,2,3}$; a Photon Sieve Telescope ${ }^{4,5}$ that has been under development at the US Air Force Academy (USAFA); the current Defense Advanced Research Projects Agency (DARPA) Membrane Optic Imager Real-Time Exploitation (MOIRE) ${ }^{6,7}$ program (illustrated in Figure 1 in an Earth surveillance application); and a Fresnel Diffractive Array Imager (FDAI) ${ }^{8,9}$ under development at Centre National d'Etudes Spatiales (CNES) in France for astrophysics. Other projects using diffractive optics as primary optical elements, particularly some based upon Cubesats, are also underway but are not discussed here.

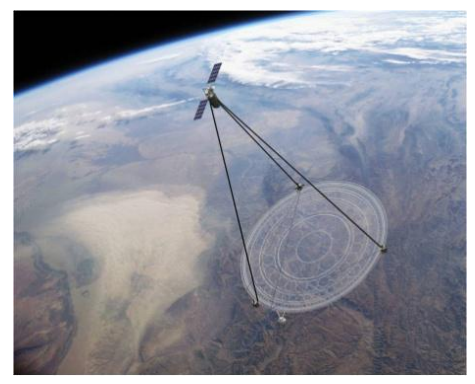

Figure 1. DARPA MOIRE concept shown conducting Earth observation

These projects have addressed a range of potential applications. Eyeglass and MOIRE were primarily directed towards observation and surveillance of the Earth, its changes, and human activities, although attention was paid to possible astronomical applications. The Photon Sieve is concerned primarily with solar physics since its low transmissivity requires a photon-rich environment, while the French FDAI concept will operate with very long integration times against astronomical targets. All could be used at least for specialized astronomical applications, but planetary observation from space (e.g., Earth or Mars) is necessarily limited by the long integration times required of concepts with low transmissivities, such as the Photon Sieve.

\footnotetext{
*howard.macewen@hmacewen.com
} 


\subsection{Principle of Operation}

All of these concepts are based upon similar principles of operation. In brief, a diffractive / refractive membrane forms an entrance aperture (the largest element in the system) that focuses incident illumination into a correcting telescope which then removes the chromatic aberration introduced by dispersion arising in the entrance aperture and corrects for some off-axis aberrations. The most significant limitation of the concept is the high degree of chromatism which only allows quality imaging in narrow bandwidths. Many applications of interest require broad-band white light illumination and thus need a (relatively) broad bandwidth for imaging. An illustration of the nominal telescope concept employed in at least two papers (Andersen ${ }^{4}$ and Atcheson ${ }^{7}$ ) is provided in Figure $2^{*}$. Other telescope concepts with broad similarities to Figure 2 include those of Meinel and Meinel ${ }^{15}$ (Figure 15), Hyde and coworkers ${ }^{1,2,3}$, and Deba ${ }^{8}$.

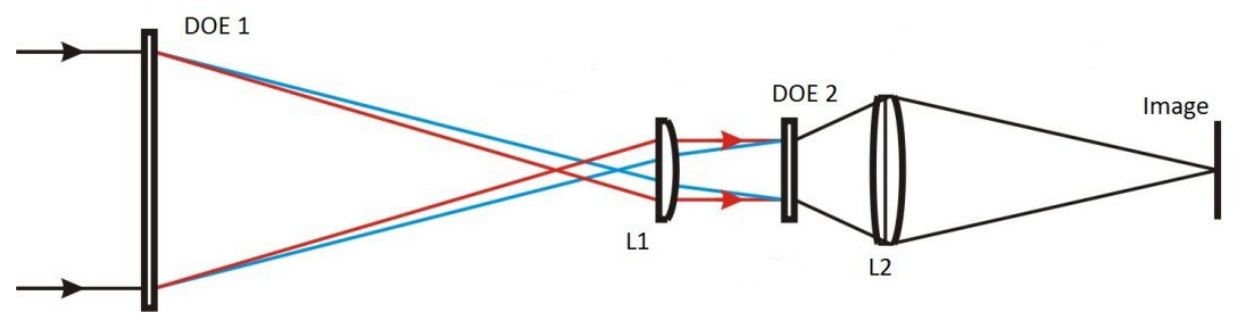

Figure 2. Nominal membrane telescope configuration (following Andersen ${ }^{4}$ )

The incident illumination ${ }^{\dagger}$ is focused by the Diffractive Optical Element 1 (DOE 1) into the entrance of the aberration corrector, which consists (in this example) of the refocusing lenses L1 and L2 and DOE 2. This second diffractive element is essentially a negative of DOE 1 and serves to correct the chromatic aberrations over the design bandwidth, which is suggested by the inclusion of both the solid and dashed ray traces that come to focus at the image plane. This is similar to a separated achromatic lens doublet. Our paper is primarily concerned with the design of DOE 1 and evaluation of the distribution of illumination around its focal point; i.e., at the entrance to the corrector. Corrector analysis will be considered in future analyses and publications.

\subsection{Applications and Implications}

If transmissive membrane telescopes can be fabricated with sufficiently wide bandwidths and high transmissivities, they will enable a wide range of defense and scientific applications, at sizes ranging from Cubesats and tactical drones to 20 meter (or greater) space telescope apertures for near real time global surveillance; spot imaging; laser detection, imaging, and tracking; and high resolution astrophysics. Rapid fabrication of "disposable" telescopes could well be enabled for a wide range of applications, defense and scientific, including ground systems, airborne telescopes (aircraft and balloons), sounding rockets, and inexpensive small satellites for tactical, commercial, and small science missions.

The technical feasibility and financial viability of such transmissive membrane telescopes is fundamentally based upon a number of characteristics of the powered entrance membrane itself. These include:

- The relative insensitivity of transmissive optics to figure errors compared to the high sensitivity of reflective optics. This arises from the fact that, for a given surface figure error, reflection introduces a much greater wavefront error than does transmission. For example, Hyde et $\mathrm{al}^{2}$ note that "A slow, thin, $\mathrm{f} / 100$ lens can tolerate 160,000 times greater shape errors than a similar mirror". This reduces the need for sub-wavelength fabrication and metrology precision for transmissive optical elements, which are very strong cost drivers for optical systems with reflective optics.

\footnotetext{
${ }^{*}$ Note that Andersen and Tullson ${ }^{5}$ replace the lens L2 with a pair of reflective elements to better correct chromatic aberrations.

${ }^{\dagger}$ Originating from infinity.
} 
- The low mass and thinness of the membranes can enable packaging and deployment approaches (for example, rolling into a cylindrical shape for launch) not available to other optical systems that must be massive and stiff. Realization of this benefit, of course, will depend upon packaging and deployment technologies that preserve the periodic structure of the membranes to the precision required.

- A thin, lightweight membrane can generally be replicated using facilities and equipment much less expensive than those required for the manufacture of reflective optical systems of equivalent capability. Facility parameters will, of course, be significantly different, in particular because of the different materials used in the membranes and the need for precision gratings as their central active elements. Fabrication of precision optical membranes can be performed using modern high speed replication processes and other high technology fabrication approaches.

Note that the benefits of membrane telescopes must be weighed against their relatively narrow bandwidths, which may require multiple collectors if a wide spectral coverage is required (conversely, of course, membrane telescopes may enable less expensive proliferation for some applications).

A few of the many applications that suggest themselves include:

- Active illumination using lasers. This application has probably the most obvious feasibility, since chromatic aberration is not a problem given the very narrow bandwidth of the illuminating laser. Uses may range from tactical surveillance (drones and balloons; low orbit satellites) to planetary / lunar cartography.

- Temporal characterization of rocket plumes in narrow bandwidths, e.g., emission lines in the near-infrared (NIR).

- Accepting bandwidth limitations, passive tactical surveillance or spot imagery from low altitudes (both airborne and spaceborne) to provide activity indications and / or high spatial resolution for identification and tracking of known targets.

- Narrow bandwidth, very high resolution imaging of astrophysical targets (possibly including specialized spectroscopy) using 20 meter class apertures to address a limited portion of the science drivers for astronomy identified in the National Academies of Science 2010 Decadal Survey ${ }^{10,11}$.

- Solar physics, such as spectroscopy in ultraviolet (UV) emission lines.

\section{STUDY OBJECTIVES}

Given the limited resources, temporal and financial, available for the study, the objectives were necessarily limited, and could include neither a complete telescope analysis and design nor an experimental verification program. Rather, they were limited to an evaluation of a possible design approach for the diffractive / refractive entrance aperture (DOE 1) and development of simplified software to rapidly generate preliminary membrane designs. The general objectives of the study were two-fold:

- Provide a simple, first order approach to defining the input illumination conditions that are produced by the entrance aperture DOE and that will require correction for image formation in the corrector element of proposed membrane telescope designs.

- Create a basis for evaluation of the precision required for fabrication and manufacturing of these membranes.

Design parameters and methodologies considered here are:

- Given a required aperture diameter, design a circular grating to focus a single design wavelength $\lambda_{0}$ at a selected focal point $\mathrm{F}_{0}$ (i.e., with a selected $\mathrm{f} / \#$ ). The design must consist essentially of a specification for the groove width, blaze angle, and depth as a function of the radial distance from the optical line-of-sight (LOS).

- Holding these parameters fixed, determine the aberrations (longitudinal and radial) that will result for off-design wavelengths $\lambda$ incident upon the lens.

- Develop a software tool (an Excel spreadsheet) that will enable rapid calculations of these membrane designs for simplified preliminary feasibility and sensitivity analyses. 


\section{METHODOLOGY}

\subsection{Design details}

The concept for the entrance aperture membrane (DOE 1 in Figure 2) combines elements of a Fresnel diffractive zone plate and a blazed grating, both illustrated in Figure 3. The zone plate (Figure 3a) has been used as a diffractive lens since the early $19^{\text {th }}$ Century, but in a stand-alone form suffers from poor transmittance for two reasons:

- First, and most obvious to inspection, is the significant fraction (approximately 50\%) of the plate that is opaque, thereby reflecting or absorbing about half of the incident illumination.

- Secondly, the plate produces multiple diffraction orders that, in the absence of a correcting mechanism, disperse a significant fraction of the incident illumination away from the detector elements of the telescope.

To address both of these issues, the Fresnel zone plate addressed in our paper's design is actually fabricated from a blazed grating (Figure 3b), using a transparent material ${ }^{*}$. This has two basic effects:

- Since the entire structure is fabricated from the transparent material, there is little loss in opaque regions (the material is not, of course, perfectly transparent, so attenuation does occur).

- The blazing refracts a significant fraction of the incident illumination into the same angle as the diffractive order chosen to meet the design requirements of the telescope.

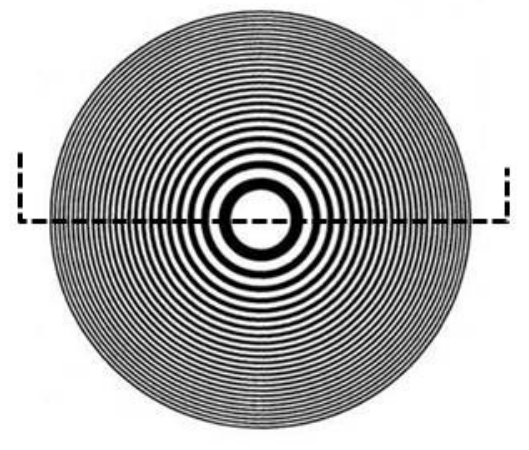

a. Fresnel Zone Plate

(Showing cross-section position)

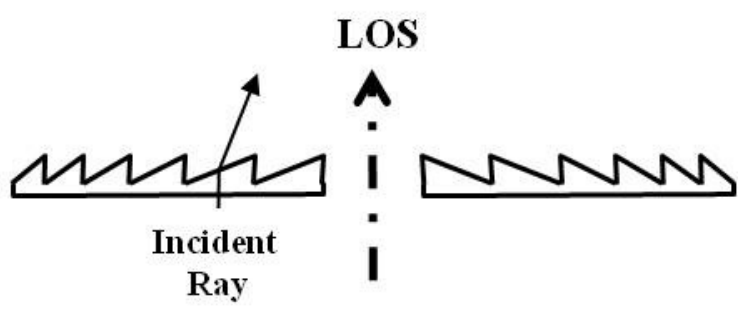

b. Blazing in cross-section

taken across Zone Plate

Figure 3. Components of membrane entrance aperture (DOE 1) Illumination incident along line-of-sight $\left(\operatorname{LOS}^{\dagger}\right)$

This section will analyze the structures shown in Figure 3 using both ray tracing (based upon the diagram in Figure 4) and wavefront analysis (Figure 6).

The detailed refractive effects of blazing will first be calculated using Figure 4, which presents a small sub-section of DOE 1. Figure 4 defines the coordinate system to be employed and illustrates the significant parameters that will result from the calculations.

\footnotetext{
${ }^{*}$ Which must be selected with the effects of the intended operating environment in mind, of course.

${ }^{\dagger}$ The LOS is taken as positive in the direction of propagation (i.e., from the object to the image).
} 


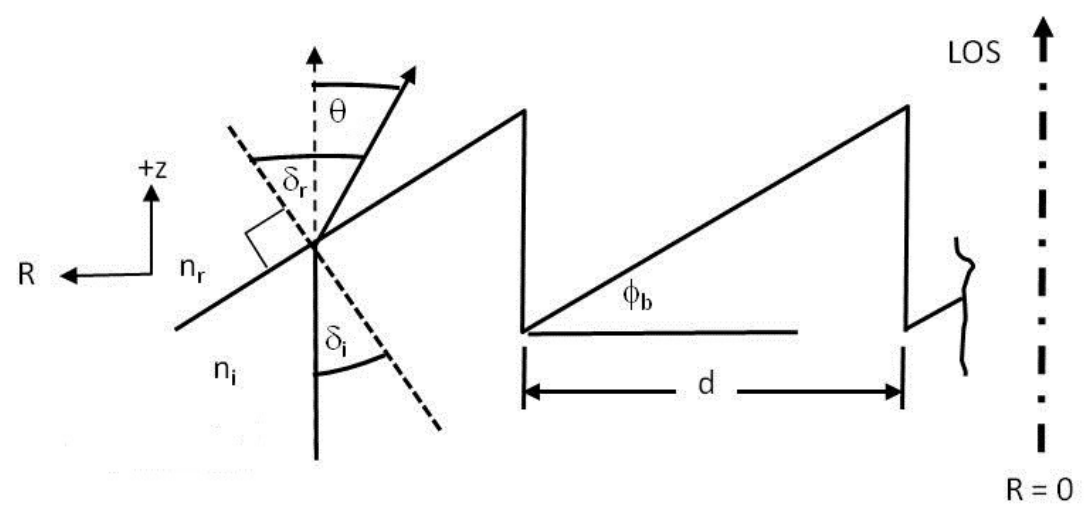

Figure 4. Detailed parameters for ray trace calculation (Detail of Figure 3b)

An external condition (the exit angle $\theta$ from the entrance aperture as a function of the radius $\mathrm{R}$ for a specific design wavelength $\lambda_{0}$ ) is imposed by the requirements of the telescope itself. As shown in Figure 5, this angle is set by the geometry of the telescope; specifically, the focal length $F_{0}$ and aperture radius $R_{0}$ (or equivalently the $f / \#$ ). Note that $R_{j}$ and $\theta_{\mathrm{j}}$ are included in the figure simply to point out that the design requires all incident rays at the design wavelength $\lambda_{0}$ to have the identical focal length $\mathrm{F}_{0}$.

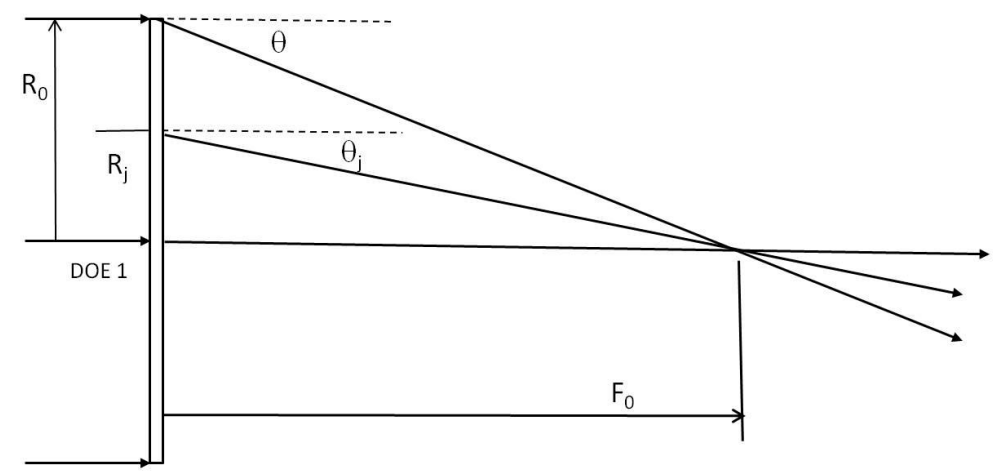

Figure 5. Telescope imposed requirements for design wavelength (Note that the exit angle $\theta$ is identical with $\theta$ in Figure 4)

\subsection{Assumptions and parameter definitions}

The principal assumptions that will hold throughout the analysis are the following, based essentially on the underlying assumption of a perfect optical system and the exclusion (for now) of manufacturing effects:

- The telescope is operating under the paraxial approximation (i.e., object space is at infinity, and off-axis illumination is not considered).

- Incident illumination is perfectly monochromatic at the design wavelength $\lambda_{0}$.

- All design wavelength incident rays are brought to a perfect focus at $\mathrm{F}_{0}$.

- The index of refraction $n_{r}$ external to the membrane is identically 1 (i.e., free space).

- The optics is free from real-world limitations and imperfections:

- Attenuation within the membrane is identically zero.

- The blazed grating faces are flat (i.e., this is not the kinoform case).

- The "vertical" faces of the grating are exactly normal to the global plane (i.e., intergroove shadowing is not considered). 
The parameters that are employed in the analysis are the following. They are graphically defined in Figures 4 and 5 above:

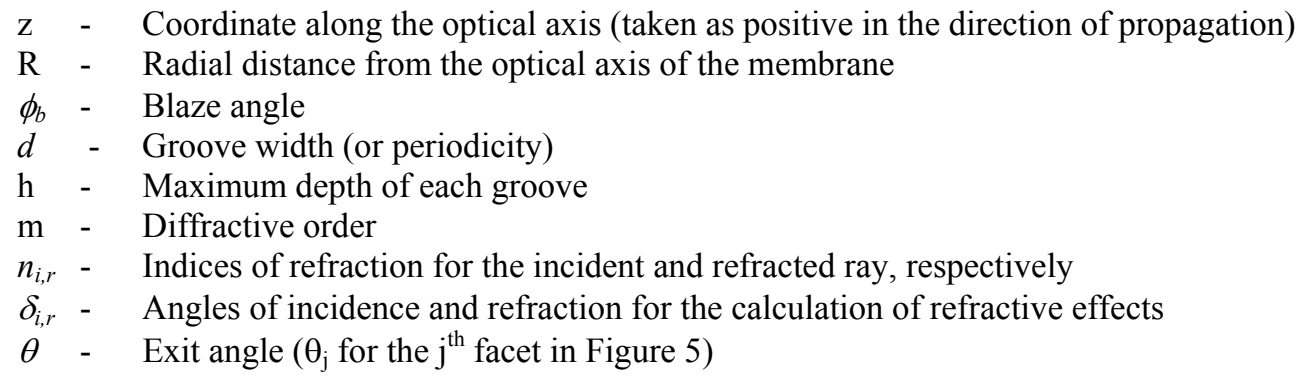

\subsection{Analysis of the design case}

This analysis will determine the dependence of the blaze angle $\phi_{\mathrm{b}}$ and the groove width $\mathrm{d}$ (and hence the groove depth $\mathrm{h}$ ) upon the radial distance of each groove from the central axis of a circular membrane, and relate these to the refractive index and the diffractive order $\mathrm{m}$. The analysis must account for a combination of refractive and diffractive effects ${ }^{12}$. As a simple, first order analysis, the work is based upon the treatments of Soskind ${ }^{13}$ and Johnson ${ }^{14}$ but includes concepts and calculations from Meinel and Meinel ${ }^{15,16}$ and O'Shea et $\mathrm{al}^{17}$.

\subsubsection{Blaze angle $\phi_{\mathrm{b}}$}

The first calculations (for the blaze angle) employs a simple ray trace approach ${ }^{*}$ in order to provide simple and intuitive insights into the effects of the entrance membrane in a telescope and provide indications of the nature and amount of achromatic correction required from other elements of the complete telescope. Beginning with Snell's law for refraction, we have:

$$
n_{i} \sin \left(\delta_{i}\right)=n_{r} \sin \left(\delta_{r}\right)
$$

From the geometry shown in Fig. $4, \delta_{i}=\phi_{b}$ and $\delta_{r}=\theta+\phi_{b}$. For the paraxial approximation and $n_{r}=1.0$ (free space refractive index), this becomes:

$$
n_{i} \sin \left(\phi_{b}\right)=\sin \left(\theta+\phi_{b}\right)
$$

Then, using simple trigonometric rearrangements and definitions of angles from Figures 4 and 5 , the blaze angle $\phi_{\mathrm{b}}$ as a function of $\mathrm{R}$ (remembering that $\mathrm{F}_{0}$ is a fixed parameter) can be expressed in terms of the telescope parameters $(\theta)$ and material properties of the membrane $\left(\mathrm{n}_{\mathrm{i}}\right)$ :

$$
\tan \left(\phi_{b}\right)=\frac{\sin (\theta)}{\left[n_{i}-\cos (\theta)\right]}=\frac{R}{\left(n_{i} \sqrt{R^{2}+F_{0}^{2}}\right)-F_{0}}
$$

\footnotetext{
${ }^{*}$ This has been validated with a wavefront (phase-matching) approach (based upon work of Johnson ${ }^{14}$ ) shown to achieve the same final results under the same conditions.
} 


$$
\emptyset_{\mathrm{b}}=\arctan \left[\frac{\mathrm{R}}{\left(\mathrm{n}_{\mathrm{i}} \sqrt{\mathrm{R}^{2}+\mathrm{F}_{0}^{2}}\right)-\mathrm{F}_{0}}\right]
$$

Calculations of the blaze angle are performed using the second form of Equation 3 (i.e., Equation $3 b$ ). Note that this approach implies that the blaze angle is a refractive phenomenon dependent upon the radial position of each diffractive ring in the blazed membrane. This is as expected, since a major purpose of blazing is to amplify the intensity for the selected diffractive order in the direction of the focal point for the design wavelength $\lambda_{0}$.

\subsubsection{Groove width $d$}

Diffractive effects are now accounted for using the grating equation as expressed in Soskind (where $\mathrm{m}$ is the diffractive order, $\lambda_{0}$ the design wavelength, and other parameters are as defined in Figures 4 and 5):

$$
n_{r} \sin (\theta)=\frac{m \lambda_{0}}{d}
$$

Since the external refractive index is identically 1 , we are left with the simple grating equation:

$$
\sin (\theta)=\frac{m \lambda_{0}}{d}
$$

Equation (5) expresses diffractive dispersion: i.e., decreasing exit angle with decreasing wavelength, given constant values for $\mathrm{m}$ and $\mathrm{d}$. Thus, based upon diffractive effects, the focal length $\mathrm{F}$ (along with the $\mathrm{fH}$ ) will decrease at longer wavelengths, as expected from long observation.

To examine the effects of diffraction in more detail, consider a wavefront approach, based upon Figure 6, in which dispersion is taken to be caused by diffraction arising from the small, finite width $\mathrm{d}$ of each facet (i.e., the equivalent of an open ring in a Fresnel zone plate).

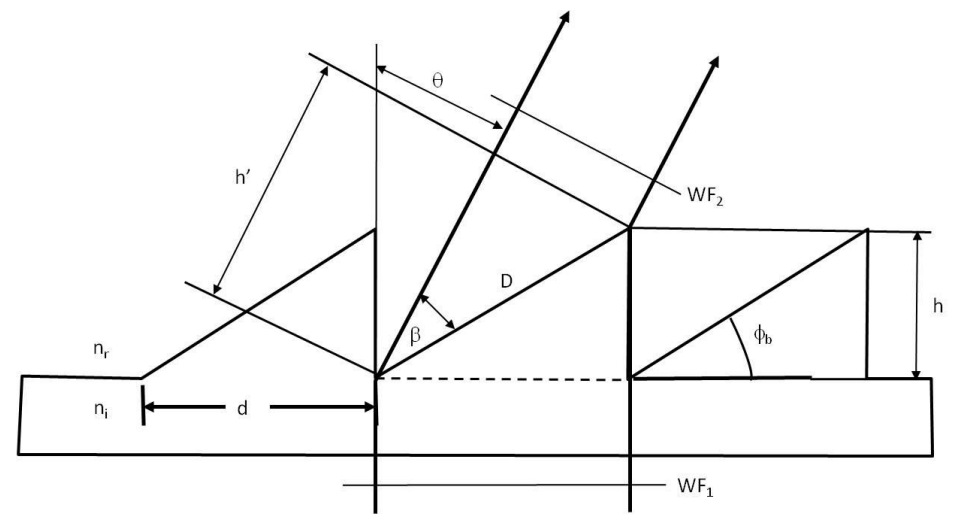

Figure 6. Wavefront based analysis

\footnotetext{
${ }^{*}$ Note that this equation is essentially equivalent to Equation (4.15) in O'Shea et $\mathrm{al}^{17}$ for the first diffractive order $(\mathrm{m}=1)$, derived in a treatment of the diffractive lens.
} 
The requirement in this case is that, assuming a constant phase across the incident wave at the location WF1, then dispersion will be such that the phase will also be constant across WF2 following dispersion by the grating. This requires that the Optical Path Differences (OPDs) (which are bounded by h and h') must differ from each other by an integral number of wavelengths of the design wavelength:

$$
\mathrm{h}^{\prime}-\mathrm{h}=\lambda_{0} \text { : or, more generally, } \mathrm{h}^{\prime}-\mathrm{h}=\mathrm{m} \lambda_{0},
$$

where $m$ is an integer that can be tentatively identified as the diffractive order. From the geometry of Figure 6 , it can be seen that:

$$
\begin{aligned}
& \mathrm{h}=\mathrm{D} \sin \left(\phi_{\mathrm{b}}\right) \\
& \mathrm{h}^{\prime}=\mathrm{D} \cos (\beta)=\mathrm{D} \cos \left[(\pi / 2)-\left(\theta+\phi_{\mathrm{b}}\right)\right]=\mathrm{D} \sin \left(\theta+\phi_{\mathrm{b}}\right)
\end{aligned}
$$

In turn, these lead directly to a modified grating equation that includes an effect from the blaze angle $\phi_{\mathrm{b}}$ :

$$
\sin \left(\theta+\phi_{\mathrm{b}}\right)-\sin \left(\phi_{\mathrm{b}}\right)=\mathrm{m} \lambda_{0} / \mathrm{D} \text { and } \mathrm{d}=\mathrm{D} \cos \left(\phi_{\mathrm{b}}\right)
$$

Here, $\mathrm{d}$ and $\mathrm{D}$ are the groove widths [d] (or periodicity when there is no space between rulings) and the facet widths [D], respectively (and defined in Figure 6).

Alternatively, since we already have Snell's law in the form $\sin \left(\theta+\phi_{\mathrm{b}}\right)=\mathrm{n}_{\mathrm{i}} \sin \left(\phi_{\mathrm{b}}\right)$, this last may be written as (in which, it should be noted, the radial position $\mathrm{R}$ and the exit angle $\theta$ are implicit in $\phi_{\mathrm{b}}$ and $\mathrm{D}$ ):

$$
\left(\mathrm{n}_{\mathrm{i}}-1\right) \sin \left(\phi_{\mathrm{b}}\right)=\mathrm{m} \lambda_{0} / \mathrm{D}=\left(\mathrm{m} \lambda_{0} / \mathrm{d}\right) \cos \left(\phi_{\mathrm{b}}\right)
$$

This immediately implies:

$$
\left(\mathrm{n}_{\mathrm{i}}-1\right) \tan \left(\phi_{\mathrm{b}}\right)=(\mathrm{m} / \mathrm{d}) \lambda_{0}
$$

Equation 9 may then be used to determine the height of the grooves $\mathrm{h}$ since, as may be seen from a simple examination of the figure:

$$
\begin{aligned}
& \tan \left(\phi_{\mathrm{b}}\right)=\mathrm{h} / \mathrm{d} \Rightarrow \mathrm{h}=\mathrm{d} \tan \left(\phi_{\mathrm{b}}\right) \\
& \mathrm{h}=\left(\mathrm{m} \lambda_{0}\right) /\left(\mathrm{n}_{\mathrm{i}}-1\right)
\end{aligned}
$$

\subsubsection{Representative numerical results - design case}

The equations determined in the above sections have been incorporated into an Excel spreadsheet to facilitate first order calculations of numerical results for membrane apertures that follow our design rules and assumptions. Input parameters required for the spreadsheet are the design wavelength, the maximum aperture radius, the focal length, the diffractive order, and the refractive index for the lens material at the design wavelength. Output parameters are the exit angle, the blaze angle, groove width, and groove height, all as functions of the radial distance from the optical axis. These initial calculations are intended to provide a baseline for additional calculations (to be discussed below) of the bandwidths and chromatic aberrations (longitudinal and radial) achievable with the baseline design. 
Calculations in the spreadsheet begin with the outermost groove and then move inward at intervals determined by the specific intentions of the operator:

- The calculations tabulated for this paper employ radii decremented by $20 \%$ of the aperture radius $\mathrm{R}_{0}$ between each step*.

- Calculations for a preliminary design could use radius decrements as small as the immediately preceding groove width; i.e., $R_{j+1}=R_{j}-d_{j}$, where it must be understood that $R_{j}$ decreases with increasing $j$, starting from $j=0$ at the outer radius.

Calculated data shown in the following was based upon input parameters chosen to replicate those used by Meinel and Meinel ${ }^{15}$ for comparative purposes. Specifically, the design wavelength $\left(\lambda_{0}\right)$ is 0.6 micrometer; the aperture radius $\left(\mathrm{R}_{0}\right)$ is 1 meter; the focal length $\left(\mathrm{F}_{0}\right)$ is 8 meters; the diffractive order is 20 ; and the index of refraction of the membrane is $1.4906^{\dagger}$ (selected for example purposes as the index of $\mathrm{PMMA}^{18}$ at a wavelength very close to the design wavelength see Table 2 below). Using these specific inputs, the entrance membrane has the characteristics in Table 1, intended to represent the beginning of the calculations for these entrance membranes:

Table 1. Representative Membrane Design

\begin{tabular}{|r|c|c|c|c|}
\cline { 4 - 5 } \multicolumn{2}{c}{} & \multicolumn{2}{c|}{ Grooves } \\
\hline Radius & $\begin{array}{c}\text { Exit Angle } \\
(\mathrm{deg})\end{array}$ & $\begin{array}{c}\text { Blaze Angle } \\
(\mathrm{deg})\end{array}$ & $\begin{array}{c}\text { Width } \\
\mathrm{d}(\mu \mathrm{m})\end{array}$ & $\begin{array}{c}\text { Height } \\
\mathrm{h}(\mu \mathrm{m})\end{array}$ \\
\hline 1 & 7.1 & 14.0 & 96.7 & 24.1 \\
\hline 0.8 & 5.7 & 11.4 & 120.6 & 24.2 \\
\hline 0.6 & 4.3 & 8.6 & 160.4 & 24.3 \\
\hline 0.4 & 2.9 & 5.8 & 240.3 & 24.4 \\
\hline 0.2 & 1.4 & 2.9 & 480.1 & 24.4 \\
\hline
\end{tabular}

The data derived from the spreadsheet is interpreted in graphical form in Figures 7 and 8 below. Figure $7^{*}$ shows two examples of exit angles and blaze angles for a diffractive order $\mathrm{m}=20$ and focal lengths $\mathrm{F}_{0}$ of 8 (baseline) and 20 meters. Figure 8 then shows calculated values for the groove width and height for a constant focal length of 8 meters but variable diffractive orders: $\mathrm{m}=10,20$, and 40 . Note the lack of variation of groove heights, which contributes to maintaining the global flatness of the membrane.

\footnotetext{
${ }^{*}$ Graphs are based upon $10 \% \mathrm{R}_{0}$ decrements for each step.

${ }^{\dagger}$ A slight difference from the 1.5 value used by Meinel and Meinel.

${ }^{*}$ Based upon telescope parameters defined in Figure 5.
} 

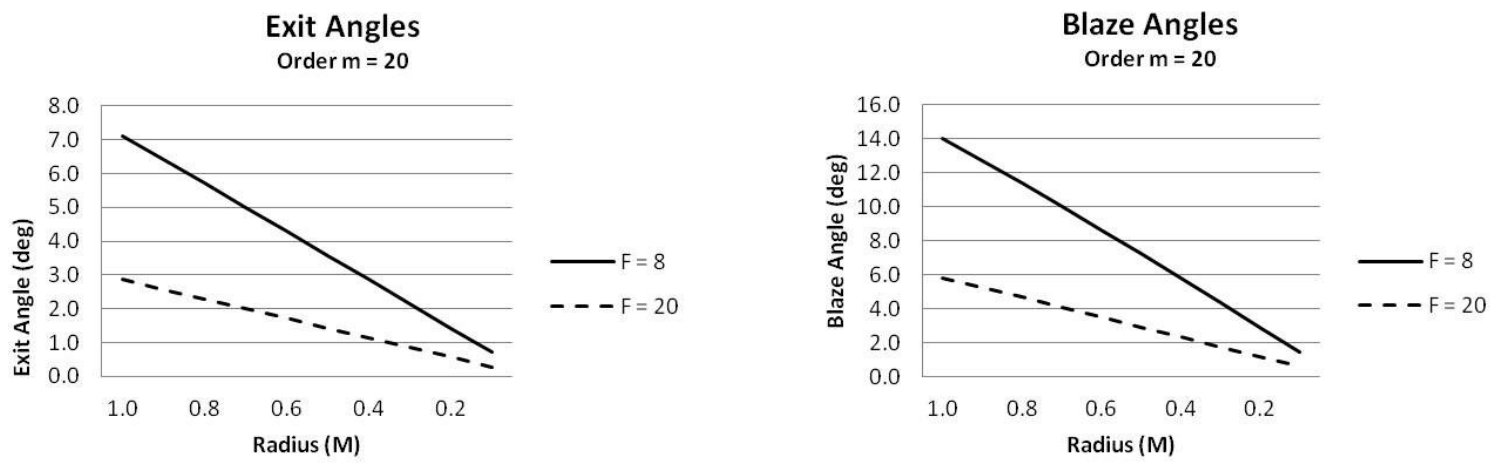

Figure 7a

Figure $7 b$

Exit angles and blaze angles for representative design conditions

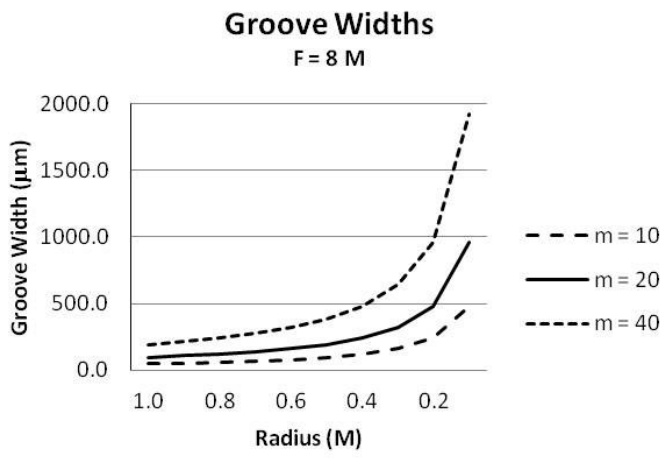

Figure 8a

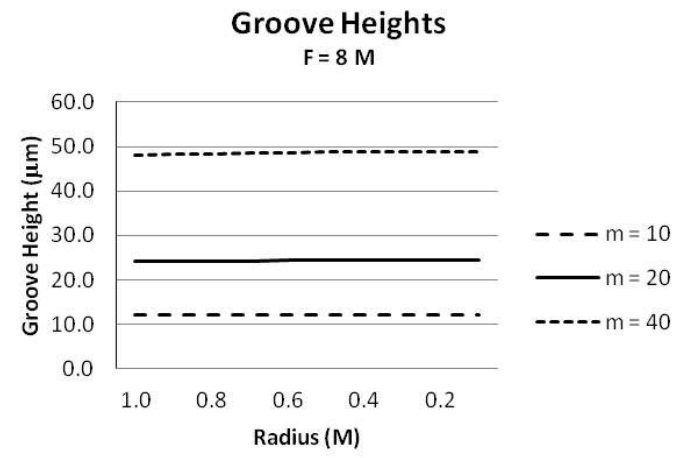

Figure $8 b$

Groove widths and heights for representative design conditions

\subsection{Chromatic effects}

The results of Section 3.3, of course, hold only for purely monochromatic illumination and in this case are valid only for a wavelength of 0.6 microns ( 600 nanometers) for a membrane material with $\mathrm{n}=1.4906$. They do, however, provide a baseline case and will now be used to evaluate conditions over a bandwidth from 0.5 to 0.7 micrometers, in terms of the focal distances (and hence longitudinal aberrations) that result over that bandwidth.

The results for the exit and blaze angles found in Figure 7 reflect only the telescope design requirements (focal length and aperture radius) and the refractive effects in the membrane, but not diffractive effects. They do, however, determine the input parameters needed to calculate diffractive effects and, in this design approach, set specific details of the grating that will determine dispersive effects (refractive and diffractive) at off-design wavelengths. Thus, this section will consider, first, the bandwidths available given refractive dispersion followed by those allowed by diffractive dispersion. Refractive dispersion is a material property (dependent upon the index of refraction), while diffractive dispersion is a geometric property (dependent upon groove widths/periodicity). The total dispersion is taken as the sum of the two once each has been calculated, although this assumption remains to be validated. 


\subsubsection{Refractive longitudinal dispersion}

Calculation of these effects depends critically upon the specific material selected. For this paper, PMMA has been selected (a known space-capable optical material), and values of $n$ that approximately cover the band from 0.5 to 0.7 microns have been employed. The values used are provided in Table 2 (extracted from Table 1 in Nikolov and Ivanov $\left.^{18}\right)$, which also includes the wavelengths used for the following calculations.

Table 2. Representative Refractive Indices for PMMA

\begin{tabular}{|c|r|r|r|r|r|}
\hline \multirow{2}{*}{$\lambda(\mu \mathrm{m})$} & 0.514 & 0.543 & 0.594 & 0.647 & 0.694 \\
\cline { 2 - 6 } & 0.500 & 0.550 & 0.600 & 0.650 & 0.700 \\
\hline $\mathrm{n}$ & 1.4945 & 1.4932 & 1.4906 & 1.4881 & 1.4864 \\
\hline
\end{tabular}

Using these values of the refractive index, Equation 2 solved for the local exit angle $\theta$ using the tangent of the blaze angle $\phi_{\mathrm{b}}$ as defined by the geometry of Figure 4, focal distances (i.e., longitudinal chromatic aberrations) for these wavelengths in the baseline case have been calculated and are provided in Table $3 \mathrm{a}$ in the Total Dispersion section below.

\subsubsection{Diffractive longitudinal dispersion}

The longitudinal aberration (i.e., focal length changes) caused by diffractive dispersion as a function of the radial position of ray incidence on the entrance aperture is calculated from the tangent of the exit angle $\theta$ determined from the grating equation (Equation 5):

$$
\mathrm{F}=\mathrm{R} \tan [\operatorname{asin}(\mathrm{m} \lambda / \mathrm{d})]
$$

It should be noted that the groove width $\mathrm{d}$ has been originally calculated from essentially the inverse of this equation, but for the design conditions (specifically, at $\lambda=\lambda_{0}$ ). For the present calculations it, along with the diffractive order $\mathrm{m}$, is held constant. The wavelength $\lambda$ and radial position $\mathrm{R}$ are the independent variables.

\subsubsection{Total longitudinal dispersion}

To the first order, the total dispersion is assumed to be the simple sum of the refractive dispersion and the diffractive dispersion. All three of these terms are presented in Table 3, which demonstrates that these two effects operate in opposite directions (as expected). However, the magnitude of diffractive dispersion is considerably greater than that of refractive dispersion, and therefore this simple summation does not seem to present a means of balancing the two and minimizing total dispersion. 
Table 3. Dispersion for the Representative Case

\begin{tabular}{|c|c|c|c|c|c|}
\hline \multirow{2}{*}{$\begin{array}{l}\text { Radius } \\
\text { (meter) }\end{array}$} & \multicolumn{5}{|c|}{ Measurement Wavelength } \\
\hline & $0.500 \mu \mathrm{m}$ & $0.550 \mu \mathrm{m}$ & $0.600 \mu \mathrm{m}$ & $0.650 \mu \mathrm{m}$ & $0.700 \mu \mathrm{m}$ \\
\hline \multicolumn{6}{|c|}{ a. REFRACTIVE FOCAL LENGTHS (meters) } \\
\hline 1 & 7.9349 & 7.9565 & 8.0000 & 8.0423 & 8.0713 \\
\hline 0.8 & 7.9356 & 7.9570 & 8.0000 & 8.0418 & 8.0705 \\
\hline 0.6 & 7.9362 & 7.9573 & 8.0000 & 8.0414 & 8.0699 \\
\hline 0.4 & 7.9366 & 7.9576 & 8.0000 & 8.0412 & 8.0694 \\
\hline 0.2 & 7.9368 & 7.9578 & 8.0000 & 8.0410 & 8.0692 \\
\hline \multicolumn{6}{|c|}{ b. DIFFRACTIVE FOCAL LENGTHS (meters) } \\
\hline 1 & 9.6229 & 8.7382 & 8.0000 & 7.3746 & 6.8378 \\
\hline 0.8 & 9.6147 & 8.7342 & 8.0000 & 7.3782 & 6.8448 \\
\hline 0.6 & 9.6082 & 8.7312 & 8.0000 & 7.3810 & 6.8502 \\
\hline 0.4 & 9.6037 & 8.7290 & 8.0000 & 7.3830 & 6.8540 \\
\hline 0.2 & 9.6009 & 8.7277 & 8.0000 & 7.3842 & 6.8564 \\
\hline \multicolumn{6}{|c|}{ c. TOTAL DISPERSION (meters) } \\
\hline 1 & 1.6880 & 0.7817 & 0 & -0.6677 & -1.2335 \\
\hline 0.8 & 1.6791 & 0.7772 & 0 & -0.6636 & -1.2257 \\
\hline 0.6 & 1.6720 & 0.7739 & 0 & -0.6604 & -1.2197 \\
\hline 0.4 & 1.6671 & 0.7714 & 0 & -0.6582 & -1.2154 \\
\hline 0.2 & 1.6641 & 0.7699 & 0 & -0.6568 & -1.2128 \\
\hline
\end{tabular}

The first two sections of Table 3 present the calculated focal lengths for the selected wavelengths for every $20 \%$ of the aperture radius, first for refractive dispersion and then for diffractive dispersion. The third section, Total Dispersion, is presented in terms of the difference between these two (refractive subtracted from diffractive), using the standard convention that the direction of propagation is taken along the $+\mathrm{z}$ axis (optical axis). Subtraction of the focal lengths is equivalent to summation of the two dispersions.

\subsection{Total radial dispersion}

Once the longitudinal aberrations have been calculated, it is a simple matter to determine the radial aberrations in the sense of the radial position (denoted by $\mathrm{r}$ ) in the paraxial image plane of DOE 1 at which each ray crosses the plane at $\mathrm{F}_{0}$, the focal point for the design wavelength. If $F(R)$ is a particular ray's focal point and $\theta(R)$ is the respective exit angle, the equation is:

$$
\mathrm{r}=\left[\mathrm{F}(\mathrm{R})-\mathrm{F}_{0}\right] \tan \theta(\mathrm{R})
$$

These values have been calculated for the same input variables as above, and the results (for the sum of refraction and diffraction: the two are not plotted separately in this case) are presented in Table 4:

\footnotetext{
${ }^{*}$ And of course the dispersion at the design wavelength of $0.6 \mu \mathrm{m}$ is identically zero.
} 
Table 4. Radial dispersion across $0.5-0.7 \mu \mathrm{m}$ bandwidth

\begin{tabular}{|c|c|c|c|c|c|}
\hline \multirow{2}{*}{$\begin{array}{c}\mathrm{R}_{0} \\
(\text { meter })\end{array}$} & $0.500 \mu \mathrm{m}$ & $0.550 \mu \mathrm{m}$ & $0.600 \mu \mathrm{m}$ & $0.650 \mu \mathrm{m}$ & $0.700 \mu \mathrm{m}$ \\
\hline \multicolumn{7}{|c|}{ TOTAL RADIAL DISPERSION (meters) } \\
\hline 1 & 0.1769 & 0.0899 & 0.0000 & -0.0901 & -0.1788 \\
\hline 0.8 & 0.1408 & 0.0716 & 0.0000 & -0.0716 & -0.1420 \\
\hline 0.6 & 0.1053 & 0.0535 & 0.0000 & -0.0534 & -0.1059 \\
\hline 0.4 & 0.0700 & 0.0355 & 0.0000 & -0.0355 & -0.0703 \\
\hline 0.2 & 0.0349 & 0.0177 & 0.0000 & -0.0177 & -0.0351 \\
\hline
\end{tabular}

\subsection{Dispersion conclusion}

For better understanding of these tables, the marginal ray from the top of the baseline diffractive lens has been plotted (not to scale) in Figure 9 below for each of the five wavelengths in the calculactions, with the ray corresponding to the design wavelength emphasized. Each ray begins at the top of the diffractive lens (DOE 1) and terminates on the right at the position determined from Tables $3 \mathrm{c}$ (for longitudinal aberration) and 4 (for radial), thereby creating a dispersion fan dependent upon the included wavelengths. Note that, as expected, the angular dispersion $(\Delta \theta)$ of shorter wavelengths (e.g., 0.5 microns) is less than that of longer wavelengths (e.g., 0.7 microns), and that diffractive dispersion is the dominant effect.

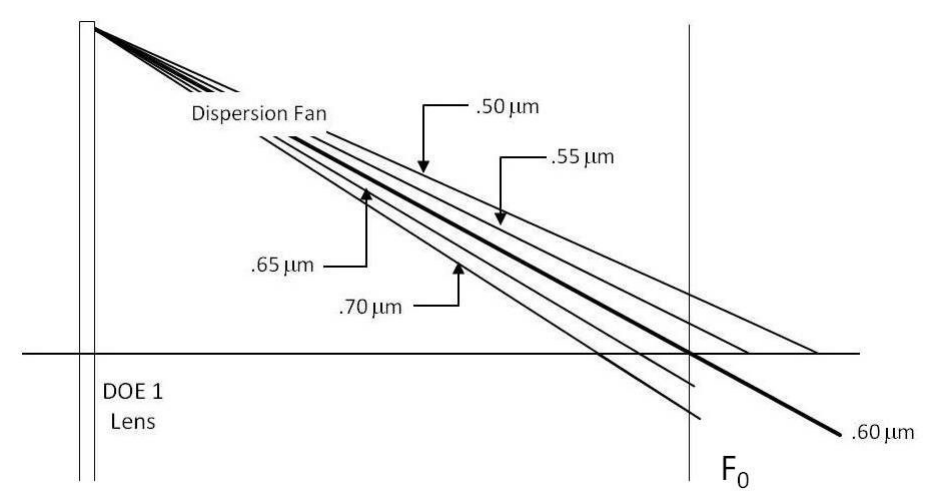

Figure 9. Illustrative ray traces for marginal ray

(Bottom half not shown)

\section{CORRECTOR CONSIDERATIONS AND OBSERVATIONS}

\subsection{Corrector Considerations}

Conceptually, ray traces such as those of Figure 9 may be used as initial conditions for preliminary design of the corrector element of a diffractive telescope. The basic approach to chromatic correction is illustrated in Figure 10, in which the dispersion fan can be easily related to the fan shown in Figure 9. Figure 10 is derived from Hyde ${ }^{1}$, who based his treatment on earlier work by Schupmann ${ }^{19}$. A quick comparison with Figure 15 in Meinel and Meinel ${ }^{15}$ will show that the basic corrector concept is also used by those authors, and the similarity with Andersen's ${ }^{4}$ work is apparent from Figure 2 of the current paper. The optics, as illustrated in Figure 10, is representational only: for example, reflective elements in the corrector optics could well be used to help minimize chromatism. 


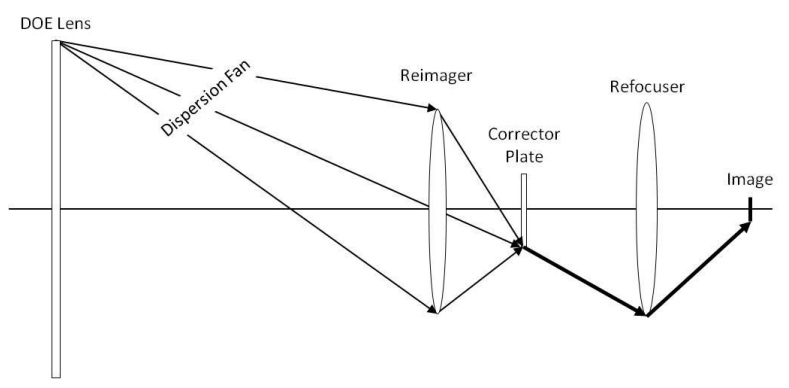

Figure 10. Representative diffractive telescope diagram

The work by Meinel and Meinel provides the best initial insights for development of corrector concepts that employ the DOE results in this paper (e.g., the chromatic dispersion fans) as starting points. An aspect common to all corrector concepts, but specifically emphasized by the Meinels, is the need for the dispersion fan to be small enough to pass fully into the entrance element of the corrector optics: i.e., the reimager in Figure 10. This will impose specific technical requirements upon the corrector elements (as well as upon DOE 1) that will be addressed in the studies planned to follow this work and to be published at later dates.

\subsection{Observations}

This analysis was intended specifically to develop the simplest, most intuitive physical insight into the optical performance of large ( 1 - 20 meters) membrane diffractive optical elements (DOEs) designed as entrance apertures for large telescopes; ground, airborne, or space. Simple spreadsheet calculations were also developed to supplement and illustrate the analysis proper.

Specific observations include the following:

- These analyses and the Excel spreadsheet based upon them are believed to provide a basis for designing and evaluating (to the first approximation) the entrance membrane of a large ${ }^{*}$, lightweight telescope based upon diffractive optics.

- A similar simple approach to first order design of the corrector elements is now required to enable intuitive evaluation of complete telescope designs, and will be the next step in this analysis.

- Detailed modeling with advanced computer models of optical systems is clearly required, since the explanatory and calculational powers of simple spreadsheets are nearing exhaustion.

- Moreover, further analysis will be necessary to determine additional factors, such as intensity distributions, diffractive efficiency, and so on that may seriously affect the conclusions that can be drawn from this work. These must be evaluated as functions of the design wavelengths and across the full wavebands required of the telescopes.

- Based upon results of these and other analyses, a serious evaluation of available materials, fabrication technologies, and manufacturing capabilities and needs for membrane lenses is required.

An early validation of the conclusions provided in this paper is essential. In particular, these conclusions are based upon the implicit assumption that refractive and diffractive dispersive effects combine through a simple summation. An experiment to verify this assumption could be accomplished at an early date with existing equipment, and could probably employ a small linear diffraction grating along with a small number (at least three) of monochromatic, continuous wave (CW) lasers to permit testing of performance across a waveband of interest. Validation with a circular grating would then be a later effort.

\footnotetext{
* Large, of course, as understood in the context of each specific mission.
} 


\section{APPENDIX: A BRIEF HISTORY}

Shortly after Thomas Young $(1804)^{20}$ discovered the wave nature of light, Augustin Fresnel $(1819)^{21}$ laid the foundation for diffractive optical elements and described what has come to be called a Fresnel zone plate or a diffractive lens. Axially symmetric diffractive lenses are discussed in detail by O'Shea, et. al. ${ }^{17}$. The theory for the diffractive or hologram optical element is well developed by Goodman $(2005)^{22}$. These optical elements are now available in relatively small sizes (less that $10 \mathrm{~cm}$ ) for commercial applications. They are regularly integrated onto rigid hologram substrate material and used in commercial camera lenses and to control laser beams.

However, the application of this technology to large (1 to 20 meters or larger) aperture deployable telescopes for highefficiency lower cost space surveillance, reconnaissance, remote sensing and astronomical imaging applications in broadband white-light is new. Hyde ${ }^{1}$ and Early ${ }^{3}$ showed that the performance of their diffractive membrane design was limited to narrow bandwidth applications.

Specifically, diffractive membrane lenses exhibit two features that limit their efficiency;

- $\quad$ The focal length of the lens (as calculated from diffractive effects) is linearly dependent on the wavelength of light, which limits the system optical bandwidth.

- $\quad$ The diffraction efficiency is low, which can yield a system transmittance as low as $1 \%$.

The new technology described in this paper promises to provide a route that can overcome these deficiencies. 


\section{REFERENCES}

[1] Hyde, R., "Eyeglass. Very large aperture diffractive telescopes," Appl. Opt., 19: 4198-4212 (1999).

[2] Hyde, R., et al, "Eyeglass: a very large aperture diffractive space telescope," Proc SPIE, Vol. 4849 (2002).

[3] Early, J., Hyde, R., and Baron, R., "Twenty meter space telescope based on diffractive Fresnel lens," Proceedings of SPIE Vol. 5166, pp. 148-156 (2004).

[4] Andersen, G; "Photon sieve telescope," UV/Optical/IR Space Telescope: Innovative Technologies and Concepts II, Howard A. MacEwen ed.; Proc. IEEE vol 5899 (2005)

[5] Andersen, G., and Tullson, D., "Broadband antihole photon sieve telescope," Appl. Opt., 46(18): 3706-3708 (2007).

[6] Blake, T., Lt Col, “MOIRE: Membrane Optic Image Real-Time Exploitation,” DARPA/TTO Briefing, Mirror Technology SBIR/STTR Workshop, 21 June 2011.

[7] Atcheson, P. et al, "MOIRE - Initial demonstration of a transmissive diffractive membrane optic for large lightweight optical telescopes," Proc. of SPIE Vol. 8442 844221-1 (2012).

[8] Deba, P., et al, "Preparing the way to space borne Fresnel Imagers: Space scenarios optical layouts," Exp. Astron. (2011) 30: 123-136.

[9] Hinglais, E., “A space Fresnel imager concept assessment study led by CNES for astrophysical applications," Exp. Astron. (2011) 30: 86-110.

[10] National Research Council, "New Worlds, New Horizons in Astronomy and Astrophysics," National Academies Press (2010).

[11] Postman, M. et al, “Advanced Technology Large-Aperture Space Telescope (ATLAST); science drivers and technology developments,” Opt. Eng. 51(1), 011007 (January 2012).

[12]Sinzinger, S. and Testorf, M., "Transition between diffractive and refractive micro-optical components," Appl. Opt. 34, 5970-5976 (1995).

[13]Soskind, Y. G., [Field guide to diffractive optics], SPIE Field Guides, Vol. FG21, SPIE Press (2011)

[14] Johnson, K. C., "Dispersion-compensated Fresnel lens," US Patent 5,161,057 (3 Nov 1992)

[15 ]Meinel, A. and Meinel, M., "Large membrane space optics: Imagery and aberrations of diffractive and holographic achromatized optical elements of high diffraction order," Opt. Eng. 41 1995-2007 (2002)

[16]Meinel, A. and Meinel, M., "Parametric dependencies of high-diffraction order achromatized aplanatic configurations that employ circular or crossed-linear diffractive optical elements," Applied Optics 41, 7155-7165 (2002) [17] O'Shea, D., et al, [Diffractive optics: design, fabrication, and test], SPIE Tutorial Texts in Optical Engineering, Vol. TT62, SPIE Press (2004)

[18] Nikolov, I. D., and Ivanov, C. D., "Optical plastic refractive measurements in the visible and the near-infrared regions," Appl. Opt., 36(13): 2067-2070 (2000).

[19] Schupmann, L., "Die medial fernrohe: eine neue konstruktion for grosse astronomisch intstrumente,” B. G. Teubner, Leipzig (1899)

[20] Young, Thomas (1804). "Bakerian Lecture: Experiments and calculations relative to physical optics," Philosophical Transactions of the Royal Society of London 94: 1-16 (1804)

[21] Fresnel, A., "Memoir on the diffraction of light presented to the French Academy of Sciences," Natura simplex et fecunda (1819).

[22] Goodman, J. W. [Introduction to Fourier Optics], Roberts and Company, Englewood CO (2005). 